\title{
Transient Numerical Assessment of Race Car Dry-Sump Oil Under Extreme Maneuvers
}

\author{
S. F. Corzo*, D. E. Ramajo and N. M. Nigro \\ CIMEC Centro de Investigación de Métodos Computacionales, UNL, CONICET, \\ FICH, Col. Ruta 168 s/n, Predio Conicet "Dr Alberto Cassano", \\ 3000 Santa Fe, Argentina. \\ *Email: santiagofcorzo@gmail.com,
}

\begin{abstract}
A numerical assessment of a dry-sump oil system was performed by Computational Fluid Dynamics (CFD). Unlike conventional cars, race cars are subjected to high accelerations that induce oil sloshing. Hence, dry-sump oil systems are required to collect the oil outside of the engine prior to be pumped inside of it again. To avoid engine malfunctions, the dry-sump must guarantee continuously oil suction in every maneuver. To perform such simulations, the model was subjected to different car maneuvers extracted from data acquisition available from real race car. It has showed that single and combined maneuvers, such as acceleration, braking and turnings can induce downward, upward and lateral accelerations higher than $2 \mathrm{~g}$ during several seconds. Therefore, four different single maneuvers (acceleration, deceleration, turn right and turn left) as well as a set of contaminated maneuvers (braking and turning) were studied. Simulations were achieved by mean of the Volume of Fluid Method (VOF) for an air-oil system. The influence of the turbulence modeling was also investigated. First a forerunner design was analyzed and both the race car tests and CFD simulations showed that for the most extreme maneuvers (pure braking and combined with braking and turning right) the original design failed before the end of the maneuvers by air suction in the pump inlet. In consequence, the dry-sump was redesigned and assessed under these extreme conditions until to ensure stable oil aspiration.
\end{abstract}

Keywords: CFD; dry-sump oil tank; multiphase flow; sloshing.

\section{INTRODUCTION}

The conventional cars collect and store the oil in the lower part of the engine, normally called oil pan or sump. The sump is attached to the bottom of the cylinder block underneath the crankcase where the crankshaft is located using a considerable spatial volume inside the hood. Sometimes baffle plates are used inside the sump to stop oil sloshing due to bouncing, rolling, and pitching of the vehicle. On the other hand, race cars are designed under the rule of saving space, reducing weight and using a layout for lowering the mass centre of the vehicle by reducing the car to floor height. For such reasons in race cars an external tank outside the engine is disposed located according to the free space given by the layout. Furthermore, the oil stored in this external tank, called dry-sump system, allows to improve the oil-air separation avoiding the foaming. The race cars are subjected to high acceleration and decelerations forces that commonly can reach values as high as $2 \mathrm{~g}$ or $3 \mathrm{~g}$ during several seconds. These forces push the oil against the 
vertical walls compromising the normal lubrication and cooling of the engine. In particular the dry-sump oil systems could guarantee oil pumping in every situation.

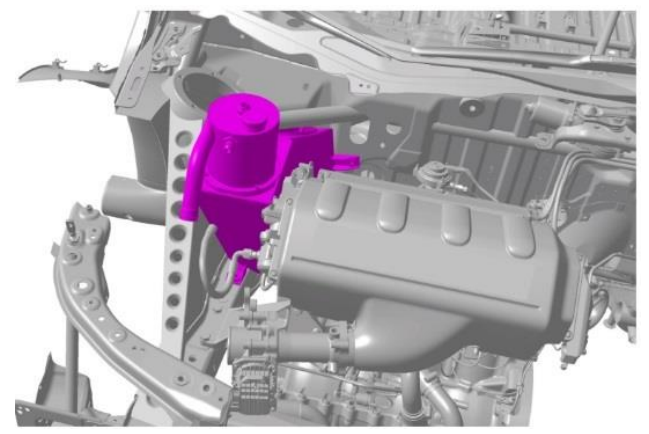

(a)

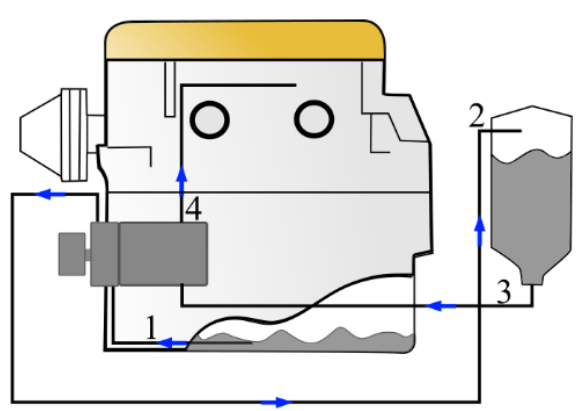

(b)

Figure 1 (a) Picture of a race engine and; (b) schematic of a dry-sump system.

Figure 1(a) shows a picture of a high-power race engine. As noted the dry-sump system, and particularly the external tank, requires large amount of volume. The Figure 1(b) displays a draw of a typical dry-sump system: the oil is collected from several points in the sump (1) by the dry-sump oil pump and then send to the top of the oil tank (2). There, the air is separated from the oil by gravity or centrifugal force and oil fall down to the bottom of the tank (3) whereas the air leaves the tank by the top towards a breather tank. Finally, the oil in the tank is suctioned again by the pump and pushed into the engine (4). The design of the dry-sump external tank must attend not to disrupt the oil pump feeding, guaranteeing the engine oil pressure over the minimum value.

In race cars, the sloshing promoted by the combination of deceleration and lateral turning could put off the oil from the suction fitting in the dry-sump tank. The fluid motion or sloshing inside tanks induced by body forces is one of the most important aspects concerning the design for maritime and road transport [1],[2]. Numerous authors have studied these phenomena by experimental [3],[4] and numerical techniques [4] focusing into avoid the loss of stability in the vehicles or the structural damage provoked by fluid motion. The most common strategies to mitigate sloshing comes from installation of baffles inside the tanks [6],[7]. The efficiency of these passive techniques has been widely reported in several applications [8],[9]. Additionally, more sophisticated ad-hoc dynamic systems have been designed for suppressing sloshing in particular industrial applications [10]. In open literature, there is not enough studies addressing with fluid motion under unsteady acceleration conditions in combined manoeuvre, such as those observed in race cars. Most of the reported studies refer to periodical or quasi-periodical conditions, by the exception of Modaressi et al. [8], who studied the influence of baffles in cylindrical tanks under quasi-uniform deceleration forces.

The present paper deals with the redesign of a dry-sump oil tank of a race car, which presented malfunction under extreme manoeuvres when the oil is pushed out of pump aspiration. In the analysed dry-sump system the external dimensions as well as the location of the inlet and outlet fittings were very restricted by the available space. Nevertheless, a very reliable design was achieved after assessing different prototypes under the most extreme manoeuvres. That was performed by means of Computational Fluid Dynamic (CFD).

The paper is outlined as; first the fluid mechanics mathematical background is presented. Then, the mesh independence and turbulence modelling are analysed by 
solving a simple geometry case. Following, the dry-sump tank model is introduced and solved for the real failure condition. Finally, the results are discussed, and the conclusion highlighted.

\section{MATHEMATICAL FORMULATION}

The mathematical background is based on the Volume of Fluid (VOF) [11] method from OpenFOAM-3.0.1 (Open Field Operation and Manipulation). The OpenFOAM code has a finite volume discretization for several types of solvers. The governing equations of the unsteady incompressible viscous flow are the Navier-Stokes equations plus the volume fraction equation [12]. The system is written as Eq. (1), (2) and (3).

$$
\begin{aligned}
& \nabla \cdot \mathrm{U}=0 \\
& \frac{\partial(\rho \mathrm{U})}{\partial \mathrm{t}}+\nabla \cdot(\rho \mathrm{UU})=-\nabla \mathrm{p}+\nabla \cdot \tau+\rho \mathrm{g}+\sigma \kappa \nabla \gamma+\mathrm{f}_{\mathrm{b}} \\
& \frac{\partial \gamma}{\partial \mathrm{t}}+\nabla \cdot\left(\mathrm{U}_{\gamma} \gamma\right)=0
\end{aligned}
$$

where $\mathrm{U}$ is the velocity field shared by the two fluids throughout the domain, called volume center velocity. $\gamma$ is the liquid phase fraction ( $\gamma=1$ for liquid and $\gamma=0$ for air), $\mathrm{U}_{\gamma}$ is the velocity of the phase represented by $\gamma, \tau$ is the deviatoric viscous stress tensor, $\rho$ is the density, $p$ is the pressure and $g$ is the gravitational acceleration. $\sigma$ is the surface tension coefficient and $\kappa$ is the local curvature of the free surface. The term $f_{b}$ represents the non-gravitational body forces. The maneuver forces of the car are included in this last. The term $\sigma \kappa \nabla \gamma$ in Eqn. 2 represents the surface tension force and was proposed by Brackbill [13]. The curvature $\mathrm{k}$ is defined as follow:

$$
\kappa=-\nabla \cdot\left(\frac{\nabla \gamma}{|\nabla \gamma|}\right)
$$

For Newtonian and incompressible fluids, the stress tensor $\mathrm{t}$ is linearly related to the strain rate tensor $\mathrm{S}, \tau=2 \mu \mathrm{S}-2 \mu(\nabla . \mathrm{U}) \mathrm{I} / 3$, with $\mathrm{I}$ the identity tensor and $\mathrm{S}=0.5\left[\nabla \mathrm{U}+(\nabla \mathrm{U})^{\mathrm{T}}\right]$. Isothermal condition was assumed for the analysis. Regarding the turbulence model, a set of simulations with real oil properties and simplified geometry with a fine enough mesh was performed using RANS (standard k-epsilon) and LES models as well as non-turbulence modeling (laminar). These tests showed that the turbulent contribution may be neglected for such refined mesh. Consequently, a laminar model was finally assumed.

The Multidimensional Universal Limiter with Explicit Solution (MULES) [14] was used to solve the volume fraction equation in Eq. (3). The PIMPLE algorithm was used for pressure-velocity coupling and the Geometric Algebraic Multi-Grid (GAMG) [16] was selected to solve the Poisson equation for the pressure. Details about the PIMPLE algorithm and its implementation in OpenFOAM have been gave by Gatin et. al.[15]. For the rest of the equations the Preconditioned Bi-Conjugate Gradient (PBICG) was chosen. First order scheme was used to discretize the divergence terms and linear scheme for the laplacian and gradient terms. For the linear solver, the convergence criteria 
at each time step was an absolute residual below 10-6 or a relative residual three orders lower than the initial. Two outer-PIMPLE iterations and two inner loop corrections were used.

\section{Validation Test}

A simple numerical test was proposed in order to assess the turbulence models and the grid refinement. A 2D partially-filled cavity was simulated considering three uniform meshes, and two turbulence models along with a laminar case. The widely used Reynolds averaged Navier-Stokes (RANS) k- $\varepsilon$ model [17] and the Large Eddy Simulation (LES) model [18] were chosen. For LES, a 3D mesh with periodic conditions was required. Both models are very used to simulate complex real geometry problems. The k-E model is based on the average of the whole length turbulent scales. On the other hand, LES simulates the large eddies, that can be represented by the grid, and models those scales that are smaller than the grid cells (subgrid scales).

The test geometry is displayed in Figure 2. A square cavity is split by a vertical partition wall, separating the square in two regions. This simple model has some similarity with the real dry-sump tank. Moreover, the side length of the square $(\mathrm{h})$, represents the longitudinal dimension of the real tank $(\mathrm{h}=0.25 \mathrm{~m})$. The partition wall has a length of $\mathrm{h} / 4$. In the initial time, the domain is filled of oil up to the middle of the partition wall height $(\mathrm{h} / 8)$. The model was perturbed with an external constant acceleration of $1 \mathrm{~g}$ suddenly applied to the liquid towards the negative $\mathrm{x}$ direction. That represents a lateral acceleration of the domain towards the positive $\mathrm{x}$ direction.

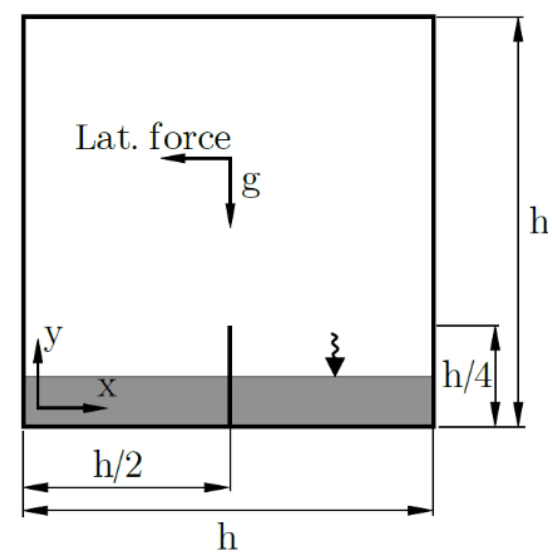

Figure 2. Test geometry.

The problem was simulated by $2.5 \mathrm{~s}$, which was enough time to get the final distribution of oil at both sides of the partition wall. At this time the liquid in both volumes has reached an almost motionlessness condition and there are not fluid overcoming the vertical wall. The coarse grid (Mesh 1) was $50 \times 50$ cells and it was refined by $\times 2, \times 4$ and $\times 8$ for the Mesh 2, Mesh 3 and Mesh 4, respectively. All meshes have uniform structured cells (hexahedrons). For LES cases, a 3D cavity of $0.25 \mathrm{~m} \times 0.25 \mathrm{~m} \times 0.1 \mathrm{~m}$ was used, and the $\mathrm{z}$ direction was discretized with the same cell size than for the other directions. Moreover, periodic boundary conditions were applied.

Table 1 shows the percentage of oil remaining in the right compartment at the end of simulation. As noted, after the maneuver more than a half of the initial oil moved into the left side. The laminar results were quite similar for all meshes except for the coarser 
one. On the other hand, the k- $\varepsilon$ model showed high dependency with the mesh, whereas for LES model it was a little. In all cases, a good agreement between the three turbulence approaches was reached for the finest mesh.

Table 1. Percentage of oil remaining in the right side at the end of simulation.

\begin{tabular}{lccc}
\hline Grid & Laminar & $\mathrm{k}-\varepsilon$ & LES \\
\hline Mesh 1 & 47.86 & 49.73 & $-^{\mathrm{a}}$ \\
Mesh 2 & 44.82 & 47.04 & 44.40 \\
Mesh 3 & 44.74 & 46.42 & 44.74 \\
Mesh 4 & 44.20 & 45.83 & 44.13 \\
\hline
\end{tabular}

${ }^{a}$ Mesh too coarse for LES modeling.

The maximum difference between the coarsest and the finest meshes was lower than $4 \%$. This means that even the coarser meshes can capture the main structures that dominates the problem. As expected, the smallest structures, such as droplets or fine ligaments, are only captured by the finest meshes but they do not noticeable change the macroscopic results. The turbulence modeling is not required to solve the problem. The RANS model shows a solution more diffusive and the flow presents more resistance to detach the tip of the partition wall.

The fact that the laminar case result was similar to the turbulence cases indicates that turbulent structures, which affect the effective viscosity, do not dominate over the inertial forces. This conclusion is in line with several papers in which free-surface problems at high Reynolds numbers were also solved with laminar models. Several researchers [19] [4], [20], [21] successfully simulated complex problems without using turbulence models finding good agreement with experimental or analytical results. Lee et al. [22] arrived at the same conclusion in Liquefied natural gas transport problems. More recently, Liu et al. [23] compared the solutions from laminar and several turbulence models finding that the first was in better agreement with experimental data.

In order to compare the solutions from the different meshes, the Figure 3 shows the oil interface for the meshes 2, 3 and 4 for the laminar and turbulent models at $t=0.2 \mathrm{~s}$ and $t=0.3 \mathrm{~s}$ respectively. At $0.2 \mathrm{~s}$, only primary structures are observed. A large wave is just overcoming the partition wall from the right to the left side and the oil in the left side is growing ups over the left wall. It can be concluded that the laminar and LES results agree very well for the three meshes. On the other hand, for the RANS model the shape of the waves crossing the partition wall differs significantly and the waves seem to be delayed with respect to the others. This would be caused by an over-estimation of the turbulent diffusion. Despite of this, the amount of oil crossing the wall at the end of simulation is similar for all cases. 


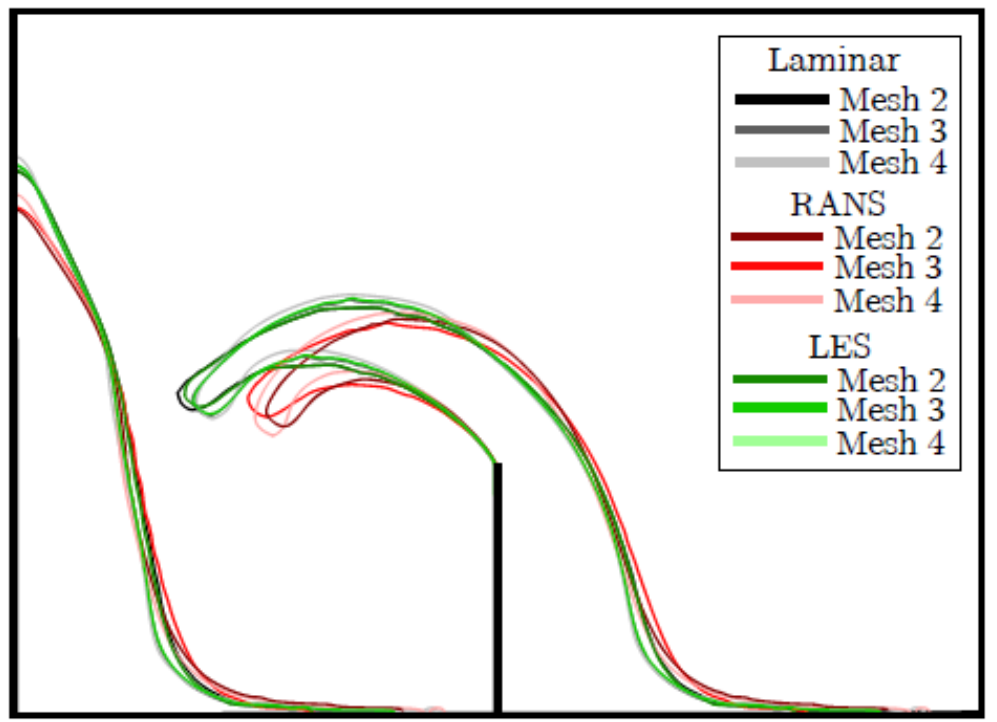

(a)
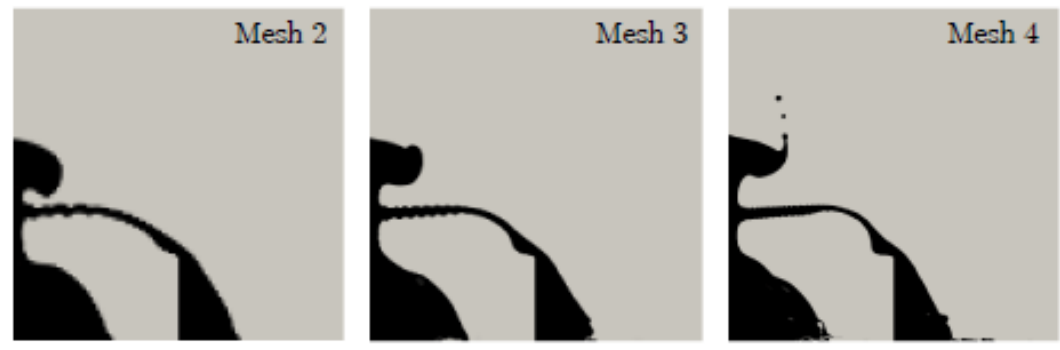

(b)

Figure 3. Mesh convergence and turbulence models assessing: (a) Oil interface at $\mathrm{t}=0.2 \mathrm{~s}$, (b) Oil interface for the laminar case and meshes 2, 3 and 4 at $\mathrm{t}=0.3 \mathrm{~s}$.

The inability of the coarser meshes to capture the secondary break-up structures does not affect the overall flow pattern. The Fig 3-b shows the results obtained for the three finest meshes and the laminar case just after the sloshing impact to the left wall $(\mathrm{t}=$ $0.3 \mathrm{~s})$. Despite that Meshes 2 and 3 do not capture the detachment of drops observed with Mesh 4, the primary oil distribution is quite similar.

On the strength of the results that have been already obtained for laminar cases, it is suitable to solve the dry-sump tank model under the same assumption. The Mesh 2 did not present large differences respect to the finest mesh. Due to that and the fact that the capture of secondary break-up structures is irrelevant for the involved problem, meshing parameters similar to Mesh 2 were adopted for the dry-sump tank.

\section{DRY-SUMP MODEL DETAILS}

The original design is shown in Figure 4(a). The dry-sump tank is located with the inlet aimed to the front of the car. That is, the tank accelerates towards the left during car acceleration maneuvers and accelerates towards the right during car deceleration maneuvers. The coordinate system in the graph indicates the direction of the forces during the four basic maneuvers.

The oil coming from the engine enters through the helical inlet in the top left side and fills the left compartment (volume 1). The air coming with the entering oil is vented 
through the central tube placed in the center of the spiral. Then, the oil flows from the left compartment to the right one (volume 2) passing through three flap valves placed at the vertical wall dividing the two compartments. The flaps work as follow: they remain partially opened under null acceleration by means of a spring mechanism allowing the free passage of oil, they open more during the acceleration of the car (towards the left) and close during deceleration maneuvers of the car. Finally, the turning maneuvers have negligible effect over the flap valves. The purpose of these valves is to avoid the passage of oil from the right to the left during deceleration maneuvers.

As shown in Figure 4(a), the bottom part of the Volume 2 is divided in two by means of a vertical baffle, which also has a flap valve. The oil suction fitting is placed at one side of the vertical baffle and the valve aims to retain the oil inside this side under lateral maneuvers. Moreover, a perforated horizontal baffle is used to avoid the oil foam to reach to the suction fitting in the lowest part of the tank, where a filter (not included in the model) is located before the outlet. Finally, the vertical tube housed in the Volume 2, is only for oil level measurement.

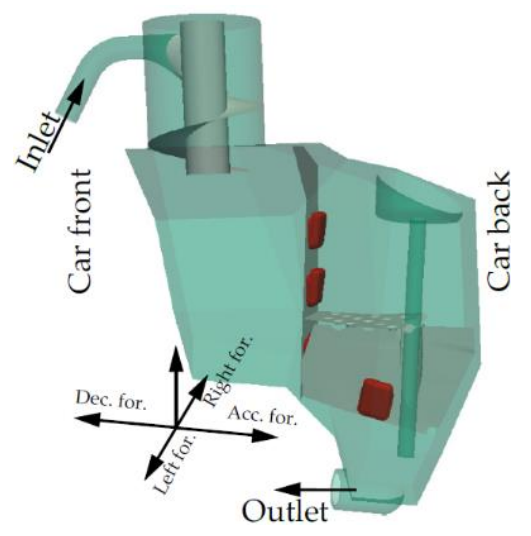

(a)

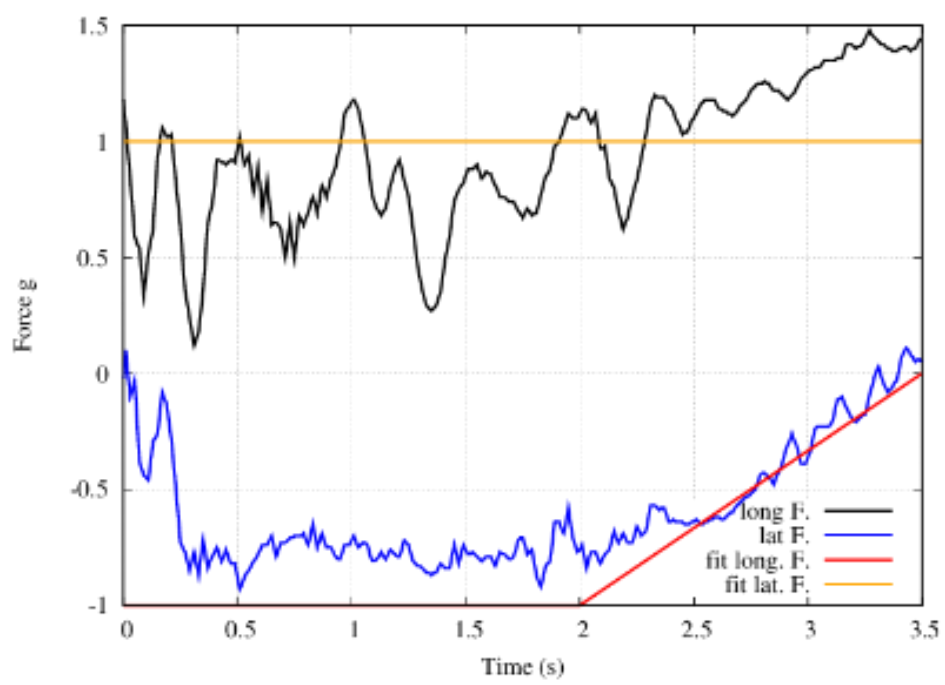

(b)

Figure 4 (a) Original design of the oil tank and; (b) a fitted friction curve.

The geometries of the original and the enhanced designs were discretized using hybrid meshes. A maximum cell size of $2.5 \mathrm{~mm}$ was adopted from the simple geometry 
test. Local refinement was required around the flat valves and the surfaces. The models were discretized with approximately 10 million of tetrahedral cells, which is large enough for the problem at hand.

A constant flow rate of 2 1/s pure oil was imposed at the inlet and the outlet, while a reference pressure was defined in the air discharge outlet in the helical zone. For these reasons, the results should be valid only after the air reaches the suction fitting outlet. The walls were defined as no-slip condition for the velocity and zero-gradient for the pressure and the phase fraction. A preliminary simulation (without accelerations) was run to achieve suitable initial conditions before to impose the maneuver. To do that, the drysump tank was initially filled with 3.51 with a constant oil level, which is right for nonaccelerated situations. Then, $0.5 \mathrm{~s}$ of simulation were required to get a developed flow in the helical inlet. After that, the transient simulation of the maneuver was started.

The force conditions were fitted from friction curves obtained from real data acquisition during track tests in which the original dry-sump system showed malfunction under extreme maneuvers and the engine stopped automatically. Figure 4(b) shows the evolution of the longitudinal and lateral forces during a combined brake and left-turning maneuver and the linear interpolations assumed for the simulation. According with the results of simulations, this maneuver was found to be the most extreme condition and was modeled by a constant lateral force of $1 \mathrm{~g}$ (right force for the fluid) joined with a constant deceleration of $1 \mathrm{~g}$ during the first $2 \mathrm{~s}$ followed by a linear reduction up to reach $0 \mathrm{~g}$ in the next $1.5 \mathrm{~s}$.

During the brake and left-turning maneuver the four flap valves were assumed completely closed, consequently the condition is truly very severe. Really both volumes are not completely isolated because the original design has a small gap between the top of the vertical baffle and the tank wall. However, due to the high position and size of the gap, oil flow is not expected to occur at least in a significant amount. The setting parameters as well as the characteristic grid size were chosen based on the validation test results.

\section{RESULTS AND DISCUSSION}

The former design was solved until air was aspirated by the suction fitting. In the real car, this situation occurred only $2 \mathrm{~s}$ before to initiate the brake left-turning maneuver. Due to that, a successful design would be achieved if oil fills the suction region along all the maneuver. Despite of that, it is important to quantify not only the oil phase fraction in there but also the remaining oil inventory in the Volume 2, which mainly feeds the suction fitting.

Figure 5(a) shows results for the brake and left turning maneuver. To have a quantitative description of the oil inventory three volumes were considered as in Figure 5(a). The Volume 1 in the left, the Volume 2 in the right and filter in the bottom representing the suction zone. In Figure 5(a), the three inventories are drawn. Note that the suction zone inventory is largely smaller than the others two and has a different scale in the graphic.

The former design quickly failed because the suction zone was abruptly emptied after $0.9 \mathrm{~s}$. Figure 5(b) shows the oil distribution for this time. Before of the first second of simulation there is still one liter of oil inside Volume 2, but it is pushed towards the vertical baffle and it accumulated over the horizontal perforated baffle. Despite the larger the orifices, the flow through them could not adequately feed the suction zone. Accordantly, the oil inventory in the suction zone fell rapidly. Even though this perforated 
plate was removed, the available oil inventory in Volume 2 would be enough to maintain the maneuver for one more second but it would be not enough to the complete the maneuver. That is because the closer valves impede the oil to pass from Volume 1 to 2. In such condition, a large amount of the total tank capacity remains unless.

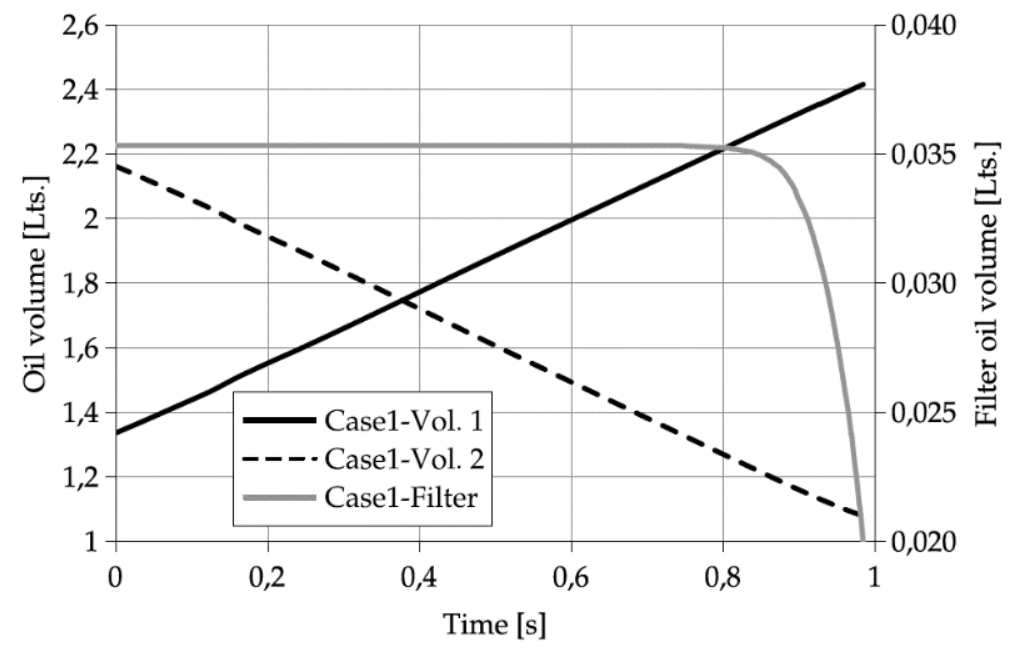

(a)

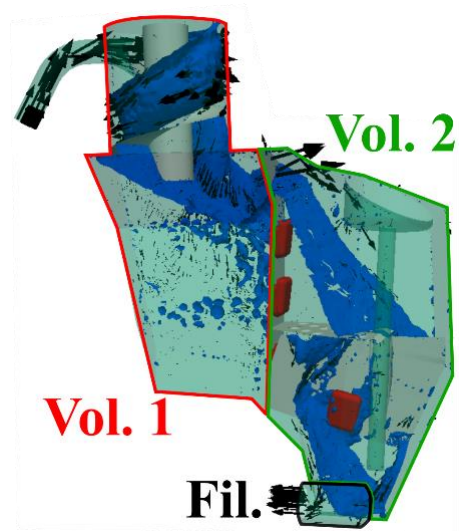

(b)

Figure 5. (a) Inventory of oil in each volume and; (b) oil interface distribution at $0.9 \mathrm{~s}$.

The race track data indicates that the car stopped by low oil pressure 3 seconds after of initiated the maneuver. The difference of time between the real and the simulated fails could be partially explained by the fact that some oil is still remaining in the oil pump and the ducts. Therefore, the measurement in oil pressure drop is delayed. As concluded, the isolation of the three volumes during this maneuver, promoted mainly by the flap valves and the baffles leads to the linear evolution of the oil inventory shown in Figure $5(\mathrm{~b})$.

Nonetheless the malfunction of the dry-sump tank under the brake and leftturning, the tank showed a good degassing performance at the helix entrance without evidence of significant amount of air in the lower part of the tank. On the other hand, the capability of the perforated baffle foam breaker is far to be simulated with the current multiphase model. 
The re-design of the tank must guarantee that air does not fill the suction zone during at least two seconds, while the entering oil supply this volume. Considering that neither the capacity and the overall shape of the tank could be increased, nor the position of the suction fitting could be put down or move to the front side, the only available possibility was to modify the inner baffles.

Attending to these constrains, two new designs were proposed. Figure 6(a) shows the first one, in which all baffles were removed (case 2). The Figure 6(b) shows the second one, which is similar to the former but putting a horizontal short blind baffle (case 3 ). The case 2 looks for having a complete communication between the two regions and to know the tank behavior without internals. On the other hand, the incorporation of the horizontal baffle in case 3 should be two functions: first to retain the oil close to the suction zone during the first time. Second, to allow the entering flow to easily pass from volume 1 to volume 2 independently of the maneuver span. The baffle is placed just below to the initial oil level with two purposes, in the short term $(\mathrm{t}<0.5 \mathrm{~s})$ should retain the initial sloshing induced by a deceleration maneuver. Next, in longer times $(t>2 s)$, it should guarantee that the entering flow spill over the baffle and takes the suction zone. The absence of mobile components, such as the flap valves, reduces the possibility of a fail by blockage of components.

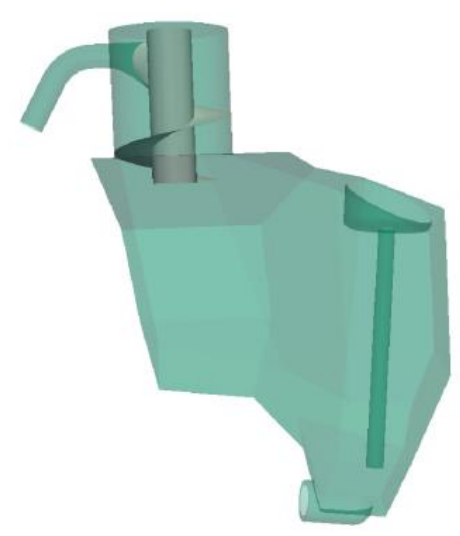

(a)

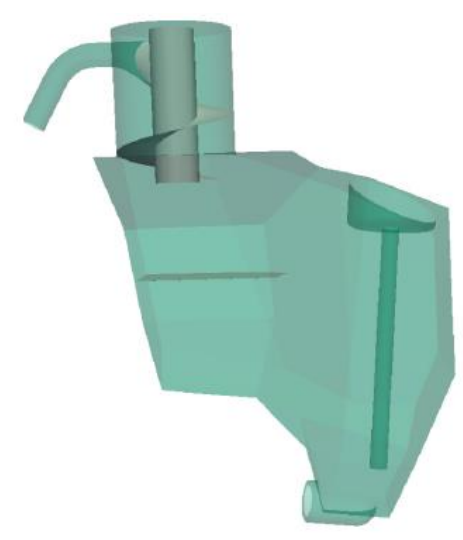

(b)

Figure 6. Proposed redesign models (a) without internals (case 2) and; (b) with a horizontal blind baffle (case 3).

This idea of mitigating the sloshing was also analyzed by other researchers, e.g. Akyildiz et al.[24] experimentally studied the effect of short horizontal baffles to break sloshing in square cavities and Zhou et al. [25] experimentally and numerically studied the effect of introduce baffles in cylindrical tanks. In both researchers a good efficiency to break the sloshing and avoid its consequences was found.

Figure 7(a) shows the inventory at the volumes 1 and 2 for both designs together with the original design (case 1). Of course, for cases 2 and 3 there is not real division between the volumes. The case without baffles (case 2) failed similar than the original design. This is due to the stream of oil ascending freely over the left wall in the Volume 1 takes oil directly from the suction zone. Therefore, the oil inventory in volume 2 falls abruptly. After $0.45 \mathrm{~s}$, the volume 2 lost more than $70 \%$ of its initial inventory, whereas the original model only had lost $18 \%$. Case 3 also shows a fast reduction on the oil inventory in volume 2 , although the curve is less abrupt. 


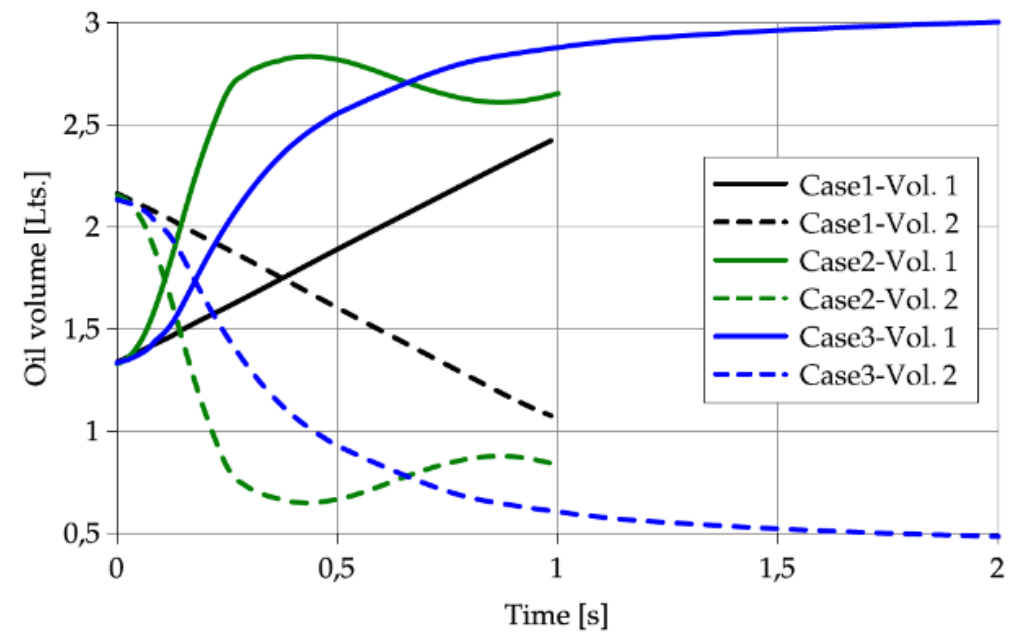

(a)

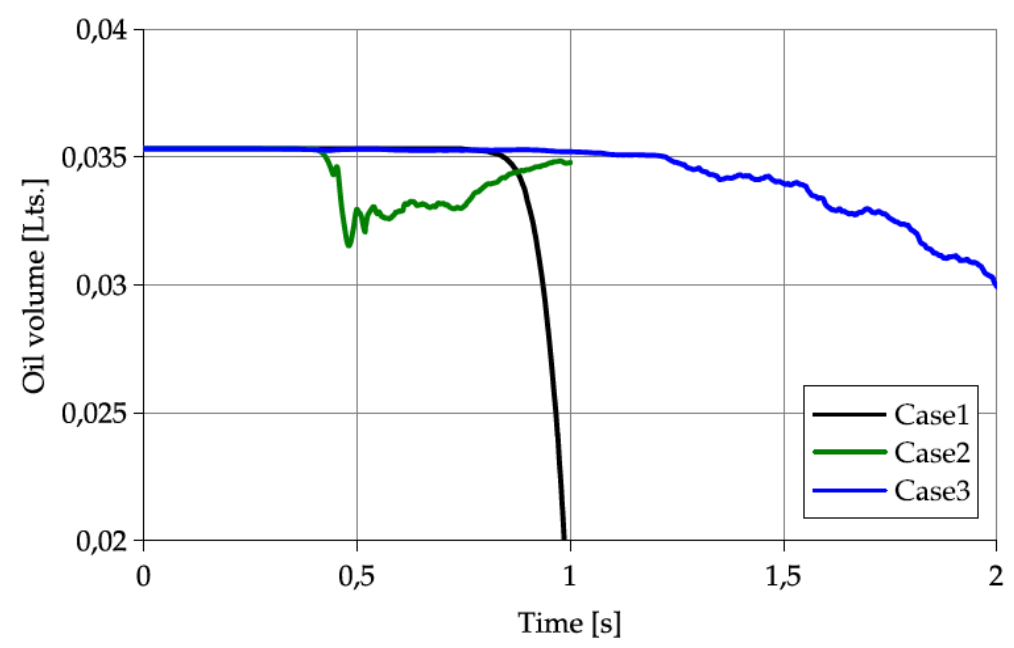

(b)

Figure 7. Oil inventory of case 1,2 and 3 for (a) overall volume monitors and; (b) filter.

Figure 7(b) shows the oil inventory in the suction zone. The case 2 fails earlier than the original design, although the oil inventory is recovery after one second. However, due to the fact that volume 2 is empty, the oil supply is no longer guarantee. This bad behavior is due to the inability for retaining the oil located close to the filter producing an inventory depletion of region 2 , which is not recovered during the first seconds.

Figure 8 compares the oil distribution for the three models and three times. The cases 1, 2 and 3 are showed in the top, middle and bottom of the figure, respectively. Regarding the case 2, after the oil fills the region $1(t=0.2 \mathrm{~s}$ approximately), a sloshing in the opposite direction is observed. Although at $\mathrm{t}=0.4 \mathrm{~s}$ this sloshing provides to the region 2, it is insufficient. Despite of this re-flow, at $t=0.4 \mathrm{~s}$ the suction fitting begins to inhale air as in Figure 7(b). From the results showed, it could be thought that the design could be acceptable if the initial emptying is avoided.

The case 3 results are showed in Figure 7 at bottom. It is observed how the lower zone of volume 1 remains quiescent and prevents the frontal sloshing. At the first time $(\mathrm{t}=0.2 \mathrm{~s})$ the imposed force causes a portion of the oil moves over the baffle towards the upper part of volume 1. However, the oil inventory around the suction zone is guaranteed. The upper zone of volume 1 accumulates oil coming from the entrance and the volume 2. 
The last occurs until reaching the balance between the body forces and mass inventory at $\mathrm{t}=0.3 \mathrm{~s}$. At $\mathrm{t}=0.4 \mathrm{~s}$ the oil begins to return from volume 1 to volume 2 . The results show a stable and maintained flow all the time. At the end of the transient the flow is stable but the safety margin is low. For this reason, the case 3 is better than the original, but it must be improved more.

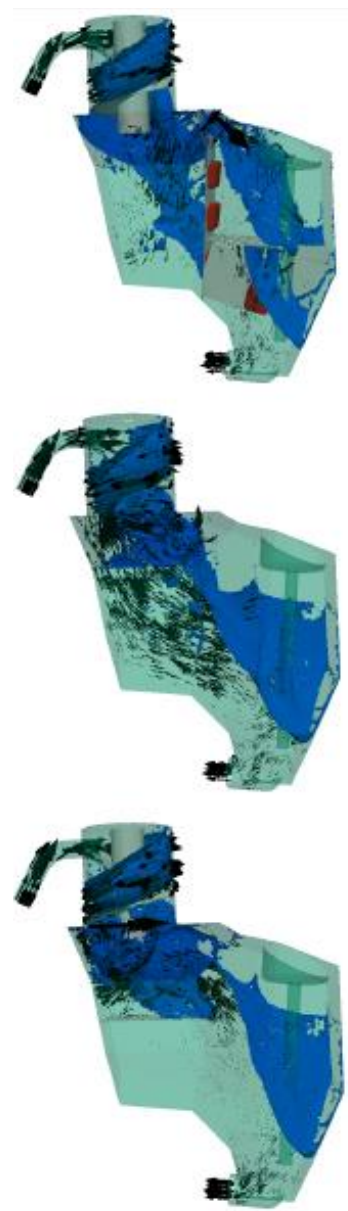

(a)
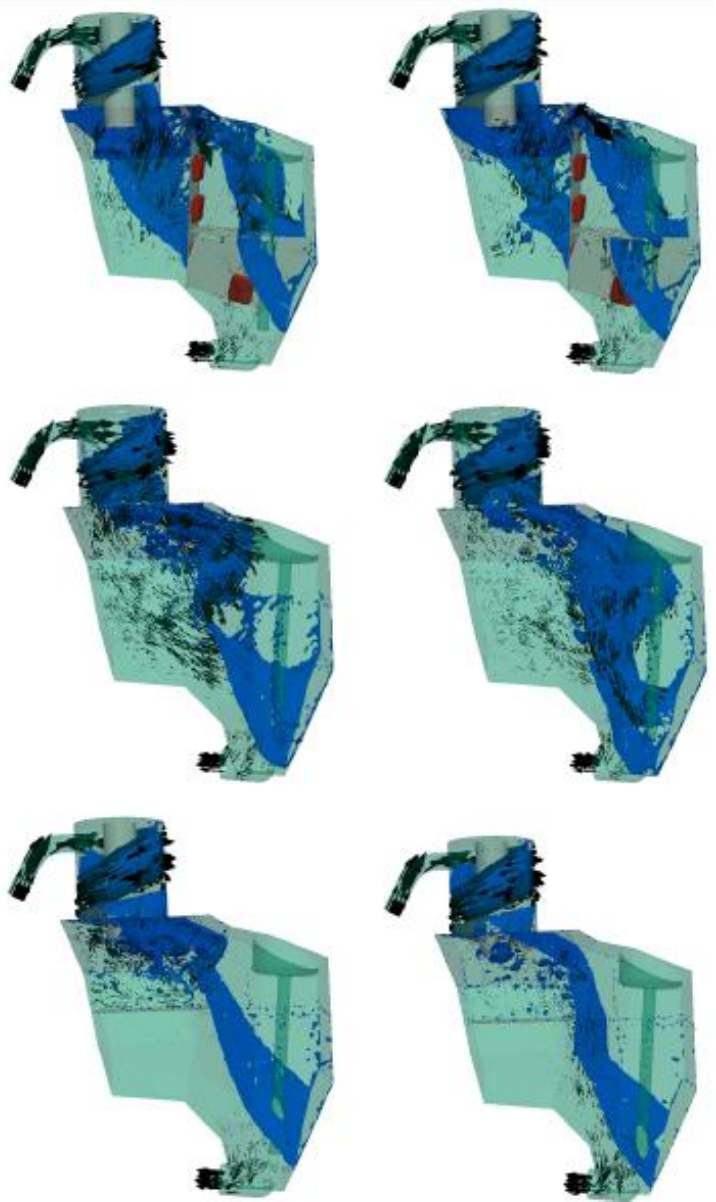

(b)

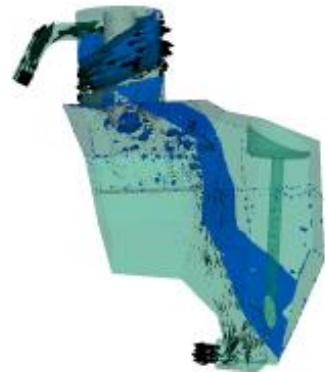

(c)

Figure 8. Oil interface distribution for case 1 (top), case 2(middle) and case 3 (bottom)

$$
\text { at (a) } \mathrm{t}=0.2 \mathrm{~s} \text {, (b) } \mathrm{t}=0.3 \mathrm{~s} \text { and (c) } \mathrm{t}=0.4 \mathrm{~s} \text {. }
$$

After $1 \mathrm{~s}$, the oil distribution in the tank shows a slow emptying of the region 2 . This proves that the mass flow from region 1 is proper. Despite the mass inventory in region 2 is lower than the original case, the redesign maintains a constant value and kept the oil in the sump region.

From the presented results, it can be concluded that the only design able to support the extremes maneuver for more than 2 seconds was the case 3 . The simulation was stopped at 2 seconds because the car acceleration starts to reduce after that time and consequently the oil-air interface begins to recover the horizontal shape. The other two designs could not maintain the oil-air interface far away from the suction zone for more than one second. Even though the case 3 reaches an almost steady interface position, it can be noted that the volume of oil close to the suction fitting remains scarce. In accordance, it is advisable to fill the tank with more oil than the original 3.5 liters. 


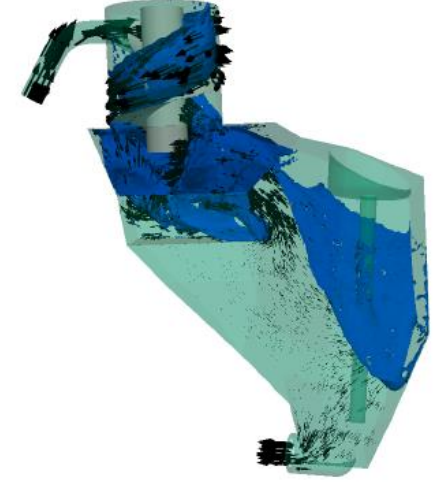

(a)

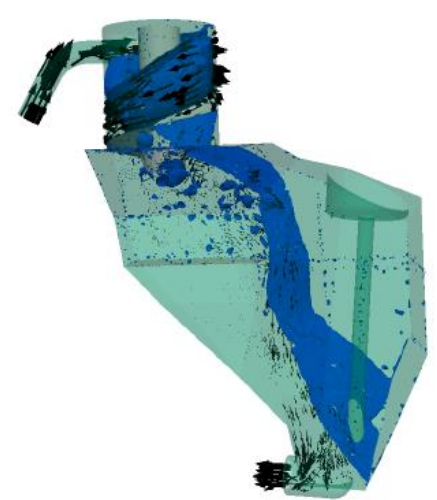

(b)

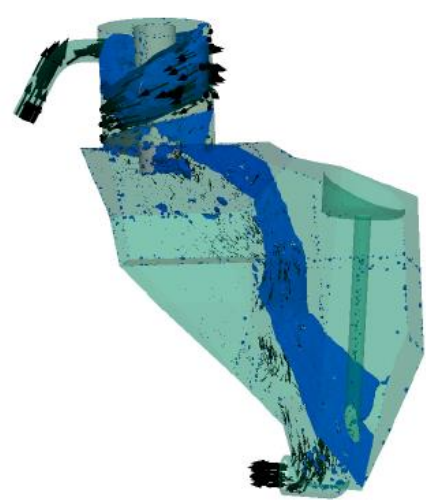

(c)

Figure 9. Oil free surface for Case 4 at (a) $t=0.2 s$, (b) $t=1.0 \mathrm{~s}$ and; (c) $t=2.0 \mathrm{~s}$.

The lower zone of volume 1 in case 3 remains quiescent and the oil located there does not contribute in any benefit to the design. Hence, a fraction of the tank could be removed to increase the oil level without increase the total amount of oil. Figure 9 shows results of the final Case 4, which was achieved by cutting a part of volume 1 . This modification reduces in 0.5 liters the volume 1 capacity but increases the oil level in 21 $\mathrm{mm}$ approximately. Note that the steady solution is reached after $\mathrm{t}=1.0 \mathrm{~s}$.

Figure 10(a) shows the comparison between cases 3 and 4 . As noted, to reduce the volume 1 has a positive impact both in the suction zone as well as in the volume 2 inventories. Figure 10(b) shows an almost constant condition is achieved after $t=1 \mathrm{~s}$. In Figure 11 a stable flow stream is established between the inlet and the suction zone. Hence, it can be concluded that the extreme braking maneuver could be longer than $2 \mathrm{~s}$ ensuring right oil suction all the time.

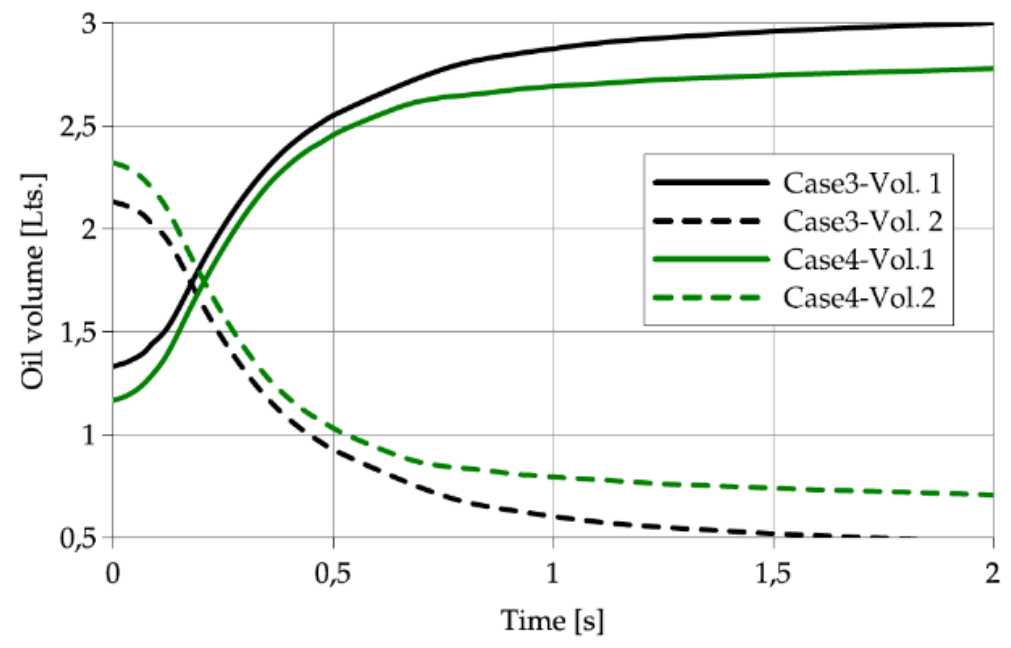

(a) 


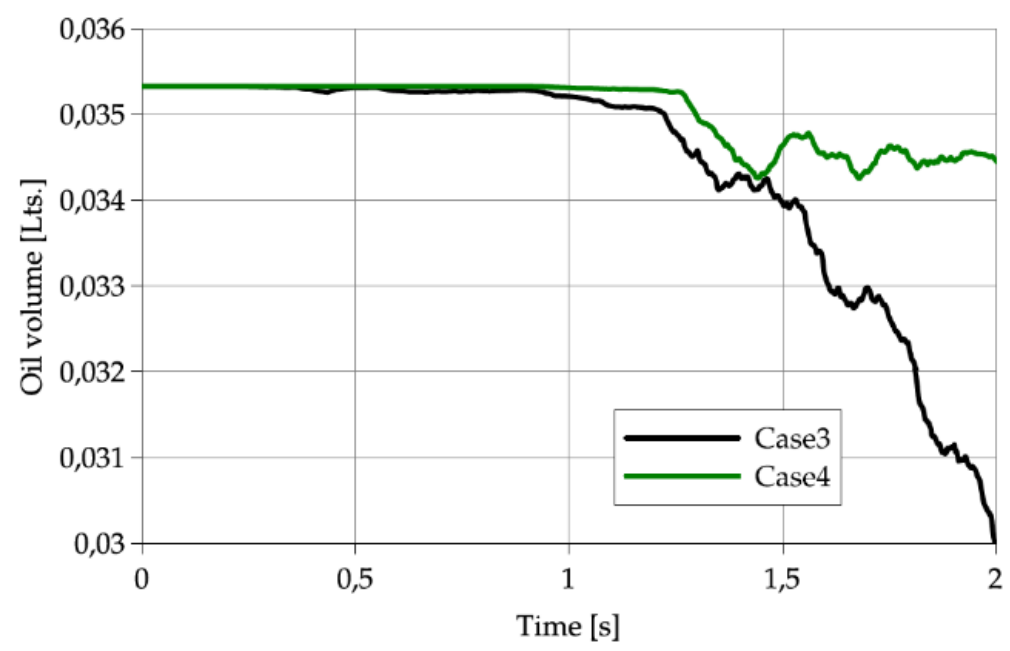

(b)

Figure 10. Oil inventory for Case 3 and 4 for (a) overall volume monitors and;

(b) filter.

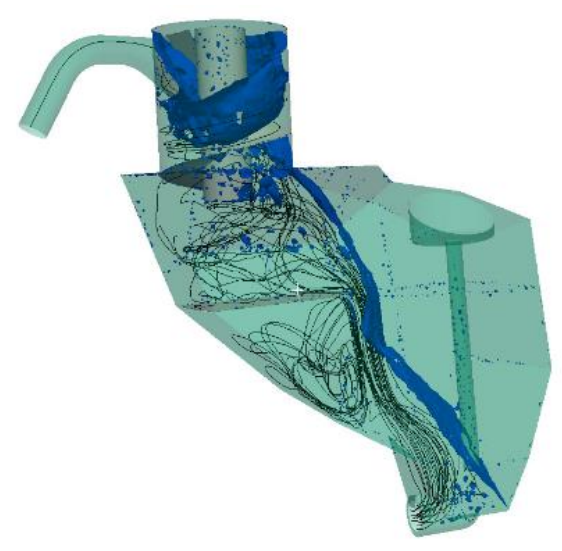

Figure 11 Oil stream-lines.

\section{CONCLUSION}

The numerical assessment of a dry-sump oil tank was performed by means of computational fluid dynamics (CFD). The volume of fluid (VOF) method was implemented to track the oil level interface along the most severe maneuver recorded in a race track. An original tank design was assessed, and the real failure behavior was also found by simulate a combined maneuver of deceleration with left turning. Two drawbacks were identified in the original tank: first, the oil around the suction zone moves far away during the first instants. Second, a direct oil stream from the inlet to the suction fitting is not guarantee.

The use of baffles and flap valves could be advantageous in some situations but also induces the isolation of the suction fitting in most of the situations. In addition, the foam breaker (horizontal perforated baffle) accumulates a large portion of the inventory over this. Therefore, a set of design modifications were proposed and numerically assessed until to reach a suitable design. The final design solves both problems by removing the baffles and flap valves, leaving only a horizontal short baffle. This allows an easy communication between the two regions, as well as retains the oil in the suction 
zone during the first second. The final design proved be able for an unlimited time. An additional advantage of the final design is in the absence of mobile parts. This avoid fails induced by valve blockage.

\section{ACKNOWLEDGEMENT}

The authors would like to thank Universidad Nacional del Litoral (CAI+D 2016-PIC 5042015 0100067-LI) and Agencia Nacional de Promocion Cientifica y Tecnologica (PICT 2013-830 and PICT 2016-2908).

\section{REFERENCES}

[1] Azadi S, Jafari A, Samadian M, Effect of tank shape on roll dynamic response of an articulated vehicle carrying liquids. International Journal of Heavy Vehicle Systems 2014; 21(3): 221-240.

[2] Hu ZQ, Wang SY, Chen G, Chai SH, Jin YT, The effects of lng-tank sloshing on the global motions of FLNG system. International Journal of Naval Architecture and Ocean Engineering 2017; 9(1): 114-125.

[3] Panigrahy Pk, Saha UK, Maity D, Experimental studies on sloshing behavior due to horizontal movement of liquids in baffled tanks. Ocean Engineering 2009; 36 (3): 213-222.

[4] Khezzar L, Seibi A, Goharzadeh A, Water sloshing in rectangular tanks-an experimental investigation \& numerical simulation. International Journal of Engineering 2009; 3(2): 174 .

[5] Kang N, Liu K, Influence of baffle position on liquid sloshing during braking and turning of a tank truck. Journal of Zhejiang University 2010; 11(5): 317-324.

[6] Ibrahim RA, Liquid sloshing dynamics: Theory and Applications. 1st ed. Cambridge University Press; 2005.

[7] Belakroum R, Kadja M, Mai TH, Maalouf C, An efficient passive technique for reducing sloshing in rectangular tanks partially filled with liquid. Mechanics Research Communications 2010; 37 (3): 341-346.

[8] Modaressi-Tehrani K, Rakheja S, Stiharu I, Three-dimensional analysis of transient slosh within a partly-filled tank equipped with baffles. Vehicle System Dynamics 2007; 45(6): 525-548.

[9] Eswaran M, Saha UK, Maity D, Effect of baffles on a partially filled cubic tank: Numerical simulation and experimental validation. Computers \& Structures 2009; 87(3): 198-205.

[10] Yano K, Higashikawa S, Terashima K, Motion control of liquid container considering an inclined transfer path. Control Engineering Practice 2002; 10(4): 465-472.

[11] Hirt CW, Nichols BD, Volume of fluid (VOF) method for the dynamics of free boundaries. Journal of computational physics 1981; 39(1): 201-225.

[12] Weller HG, A new approach to VOF-based interface capturing methods for incompressible and compressible flow. Open-CFD Technical Paper; 2008.

[13] Brackbill JU, Kothe DB, Zemach C, A continuum method for modeling surface tension. Journal of computational physics 1992; 100(2): 335-354.

[14] Zalesak ST, Fully multidimensional flux-corrected transport algorithms for fluids. Journal of computational physics 1979; 31(3): 335-362. 
[15] Gatin I, Vukčevic V, Jasak H, Rusche H, Enhanced coupling of solid body motion and fluid flow in finite volume framework. Ocean engineering 2017; 143(1): 295304.

[16] Bramble J, Zhang X, The analysis of multigrid methods. Handbook of numerical analysis 2000; 7(1): 173-415.

[17] Wilcox D, Turbulence Modeling for CFD. 3rd ed. DCW Industries; 1998.

[18] Smagorinsky J, General Circulation Experiments with the Primitive Equations. Monthly Weather Review 1963; 91(3): 99-164.

[19] Kelecy FJ, Pletcher RH, The development of a free surface capturing approach for multidimensional free surface flows in closed containers. Journal of computational physics 1997; 138(2): 939-980.

[20] Alves MVC, Barbosa JR, Prata AT., Analytical and CFD modeling of the fluid flow in an eccentric-tube centrifugal oil pump for hermetic compressors. International Journal of Refrigeration 2013; 36(7): 1905-1915.

[21] Koh CG, Luo M, Gao M, Bai W, Modelling of liquid sloshing with constrained floating baffle. Computers \& Structures 2013; 122(1): 270-279.

[22] Lee DH, Kim MH, Kwon SH, Kim JW, Lee YB, A parametric sensitivity study on LNG tank sloshing loads by numerical simulations. Ocean Engineering 2007; 34(1): 3-9.

[23] Liu D, Tang W, Wang J, Xue H, Wang K, Comparison of laminar model, RANS, LES and VLES for simulation of liquid sloshing. Applied Ocean Research 2016; 59(1): 638-649.

[24] Akyildiz H, Unal E, Experimental investigation of pressure distribution on a rectangular tank due to the liquid sloshing. Ocean Engineering 2005; 32(11): 1503-1516.

[25] Zhou R, Vergalla M, Chintalapati S, Kirk D, Gutierrez H, Experimental and numerical investigation of liquid slosh behavior using ground-based platforms. Journal of Spacecraft and Rockets 2012; 49(6): 1194-1204. 